
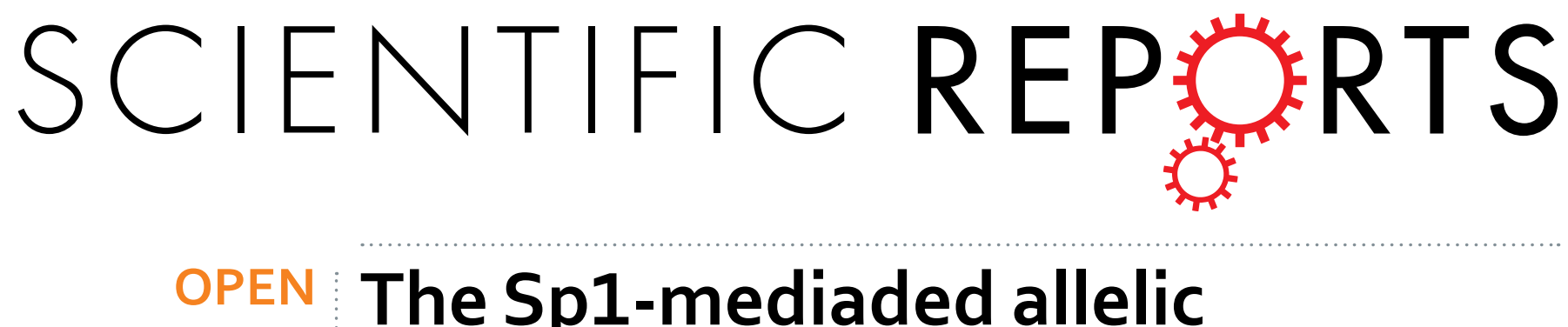

\title{
The Sp1-mediaded allelic regulation of MMP13 expression by an ESCC susceptibility SNP
}

Received: 30 October 2015

Accepted: 12 May 2016

Published: 01 June 2016

\section{rs2252070}

Meng Shi ${ }^{1,2, *}$, Jianhong Xia ${ }^{3, *}$, Huaixin Xing ${ }^{4, *}$, Wenjun Yang ${ }^{5}$, Xiangyu Xiong ${ }^{2}$, Wenting Pan ${ }^{2}$, Sichong Han ${ }^{2}$, Jinhua Shang ${ }^{2}$, Changchun Zhou ${ }^{6}$, Liqing Zhou ${ }^{3} \&$ Ming Yang $^{1}$

Metallopeptidase 13 (MMP13), a well-known and highly regulated zinc-dependent MMP collagenase, plays a crucial part in development and progression of esophageal squamous cell carcinoma (ESCC). Therefore, we examined associations between ESCC susceptibility and four haplotype-tagging single nucleotide polymorphisms (htSNPs) using a two stage case-control strategy. Odds ratios (OR) and $95 \%$ confidence intervals $(95 \% \mathrm{Cl})$ were computed by logistic regression model. After analyzing $1588 \mathrm{ESCC}$ patients and frequency-matched 1600 unaffected controls, we found that MMP13 rs2252070 G > A genetic polymorphism is significantly associated with ESCC risk in Chinese Han populations (GA: OR $=0.63,95 \% \mathrm{Cl}=0.54-0.74, P=1.7 \times 10^{-6}, \mathrm{AA}: \mathrm{OR}=0.73,95 \% \mathrm{Cl}=0.66-0.81, P=1.8 \times 10^{-6}$ ). Interestingly, the rs2252070 G-to-A change was shown to diminish a Sp1-binding site in ESCC cells. Reporter gene assays indicated that the rs2252070 A allele locating in a potential MMP13 promoter has low promoter activities. After measuring MMP13 gene expression in sixty-six pairs of esophageal cancer and normal tissues, we observed that the rs $2252070 \mathrm{~A}$ protective allele carriers showed decreased oncogene MMP13 expression. Results of these analyses underline the support of the notion that MMP13 might function as a key oncogene in esophageal carcinogenesis.

Esophageal squamous cell carcinoma (ESCC) is one of the most common malignant tumors in the world, showing a relatively high morbidity in China ${ }^{1}$. Overall, ESCC patients have poor prognosis unless exhaustive treatments, including radical surgery, chemotherapy, and radiotherapy, are given. Accumulated evidences indicate that cigarette smoking, heavy ethanol consumption, micronutrient deficiency as well as dietary carcinogen exposure are main environmental risk factors of ESCC ${ }^{2,3}$. Recent progress on genome-wide association studies (GWAS) and candidate gene studies indicate that genetic makeup also contributes to ESCC etiology ${ }^{4-10}$.

As a family of zinc-ion dependent endopeptidases, matrix metalloproteinases (MMPs) comprise more than 21 subtypes $^{11,12}$. MMPs include collagenases and gelatinases, which mediate degradation of basement membranes and the extracellular matrix ${ }^{11,12}$. Matrix metalloproteinases 13 (MMP13), encoded by the MMP13 gene, is a well-known and highly regulated zinc-dependent MMP collagenase ${ }^{11}$. It has been reported that MMP13 is significantly overexpressed in ESCC tissues compared with normal esophageal epithelium ${ }^{12}$. Deregulated $M M P 13$ expression might impact prognosis of ESCC patients through tumor invasion, vascular permeation, and lymph node metastasis ${ }^{13-15}$. MMP13, in combination with MMP7 and MMP9, are involved in early stage development of ESCC, and their co-expression predicts poor outcome for relatively early stage ESCC cases ${ }^{14}$. Moreover, MUC1

${ }^{1}$ Shandong Key Laboratory of Radiation Oncology, Cancer Research Center, Shandong Cancer Hospital affiliated to Shandong University, Shandong Academy of Medical Sciences, Jinan, Shandong Province, China. ${ }^{2}$ Beijing Laboratory of Biomedical Materials, College of Life Science and Technology, Beijing University of Chemical Technology, Beijing, China. ${ }^{3}$ Department of Radiation Oncology, Huaian No. 2 Hospital, Huaian, Jiangsu Province, China. ${ }^{4}$ Department of Anesthesiology, Shandong Cancer Hospital and Institute, Jinan, Shandong Province, China. ${ }^{5}$ Key Laboratory of Fertility Preservation and Maintenance (Ministry of Education), Ningxia Medical University, Yinchuan, Ningxia, China. ${ }^{6}$ Clinical Laboratory, Shandong Cancer Hospital affiliated to Shandong University, Shandong Academy of Medical Sciences, Jinan, Shandong Province, China. *These authors contributed equally to this work. Correspondence and requests for materials should be addressed to L.Z. (email: zlq-hill@163.com) or M.Y. (email: aaryoung@yeah.net) 
induces cancer cell metastasis by upregulating MMP13 in $\mathrm{ESCC}^{15}$. Due to the crucial role of MMP13 in ESCC development, it is essential to figure out the molecular mechanisms in fine-regulation of $M M P 13$ expression during tumorigenesis.

In the MMP13 gene locus, multiple functional single nucleotide polymorphisms (SNPs) have been identified. One of the most studied SNPs is the MMP13 rs2252070 polymorphism. Yoon et al. firstly evaluated its biological function via in vitro reporter gene assays and electrophoretic mobility-shift assays (EMSA) and showed that this polymorphism was a functional variant ${ }^{16}$. In HepG2 cells, the $M M P 13$ promoter with the rs2252070 A allele had approximately twice as much transcriptional activity as that with the Gallele in the same position $(P=0.0037)^{16}$. EMSA using nuclear extracts prepared HepG2 cells demonstrated that $M M P 13$ probes containing the A allele differed in their binding to nuclear factors from the probes containing the $\mathrm{G}$ allele ${ }^{16}$. The associations between this functional SNP and multiple malignancies were repeatedly investigated in different ethnic populations ${ }^{17-20}$. However, the details on how this genetic variant impacts $M M P 13$ expression is still largely unknown. Considering the importance of MMP13 in cancer development, we selected 4 haplotype-tagging SNPs (htSNP) across the whole MMP13 locus and conducted three large independent hospital-based case-control studies to investigate the association between MMP13 genotypes and ESCC risk. In addition, to the best of our knowledge, we firstly examined the fine-regulation of MMP13 expression by rs2252070-mediaed allelic binding of Sp1 and its involvement in ESCC. To validate the biological function of MMP13 rs2252070 genetic variant in vivo, we detected the association between its genotypes and MMP13 mRNA expression levels in normal and cancerous esophagus tissues.

\section{Materials and Methods}

Study subjects. This study consisted of two case-control sets: (a) Jiangsu set: 588 ESCC cases from Huaian No. 2 Hospital (Huaian, Jiangsu Province, China) and sex- and age-matched 600 controls. (b) Shandong set: 1000 cases with ESCC from Shandong Cancer Hospital affiliated to Shandong University, Shandong Academy of Medical Sciences (Jinan, Shandong Province, China) and sex- and age-matched ( \pm 5 years) 1000 healthy controls. Sixty-six pairs of ESCC specimens and esophagus normal tissues adjacent to the tumors were obtained from surgically removed specimens of patients in Bethune International Peace Hospital and Huaian No. 2 Hospital. All individuals were ethnic Han Chinese. At recruitment, the informed consent was obtained from each subject. The detailed information on subject recruitments can be found in Supplementary Table 1 and our previous studies $^{21}$. This study was approved by the Institutional Review Boards of Huaian No. 2 Hospital and Shandong Cancer Hospital affiliated to Shandong University, Shandong Academy of Medical Sciences. At recruitment, the written informed consent was obtained from each subject. The methods were carried out in accordance with the approved guidelines.

SNP selection and genotyping. There are multiple SNPs in MMP13 covering a $\sim 13 \mathrm{~kb}$ region of chromosome 11q22.3. As a result, we utilized an htSNP approach to examine the $M M P 13$ polymorphisms globally ${ }^{22}$. Genotyped HapMap SNPs among Han Chinese population (HCB data, HapMap Rel 27, NCBI B36) with a minor allele frequency $>5 \%$ were included in the selection. The htSNPs were chosen in a $\sim 17 \mathrm{~kb}$ region $(\sim 13 \mathrm{~kb} M M P 13$ locus and $2 \mathrm{~kb}$ up-stream as well as $2 \mathrm{~kb}$ down-stream regions of MMP13). Four htSNPs were finally selected with Haploview 4.2 software on a block-by-block basis, using a method described previously with the sample size inflation factor, $\mathrm{Rh}^{2}$, of $\geq 0.8$ (Supplementary Table 2). MMP13 htSNPs (rs11225490, rs2252070, rs17099788 and rs3758854) were analyzed by the MassArray system (Sequenom Inc., San Diego, California, USA). A 5\% blind, random sample of study subjects was genotyped in duplicates and the reproducibility was $99 \%$.

EMSA. Synthetic double-stranded and 3' biotin-labeled oligonucleotides corresponding to the Sp1 consensus binding sequence, $M M P 13$ rs2252070G or rs2252070A sequences (Supplementary Table 3) and pure recombinant Sp1 protein (E639A, Promega) were incubated at $25^{\circ} \mathrm{C}$ for 20 min using the Light Shift Chemiluminescent EMSA Kit (Pierce, Rockford, IL). The reaction mixture was separated on 6\% PAGE, and the products were detected by Stabilized Streptavidin-Horseradish Peroxidase Conjugate (Pierce). Unlabeled probes at 100-fold molar excess were added to the reaction mixture before the addition of biotin-labeled probes in competition assays.

MMP13 reporter gene constructs. Specific primer pairs (Supplementary Table 4) with XhoI and KpnI restriction sites were used to amplify multiple deletion fragments spanning $5^{\prime}$-region of $M M P 13$ (from $-186 \mathrm{bp}$ to $-41 \mathrm{bp}$, relative to the transcription start site) from human genomic DNA carrying MMP13 rs2252070GG or rs2252070AA genotype. The PCR products were then digested with XhoI and KpnI (New England Biolabs) and ligated into an appropriately digested pGL3-Basic vector (Promega) containing the firefly luciferase gene as a reporter. The resultant plasmid, designated pMMP-G or pMMP-A. Complete DNA sequencing confirmed the orientation and integrity of these two reporter constructs.

Dual luciferase reporter assays. KYSE30 and KYSE150 ESCC cells were transfected with both reporter constructs (pGL3-Basic, pMMP-G or pMMP-A) and pRL-SV40 (Luciferase Assay System; Promega). Dual luciferase activities were examined at $48 \mathrm{~h}$ after transfection as previously described ${ }^{23,24}$. For each reporter construct, three independent transfections were done, and each was performed in triplicate.

Real-time Analysis of MMP13 mRNA. Total RNA samples from esophageal tissue specimens were extracted using TRIzol Reagent (Invitrogen) and converted to cDNA using the ReverTra Ace qPCR RT Kit (TOYOBO). MMP13 mRNA expression was detected using the SYBR-Green real-time quantity PCR method as described previously ${ }^{23,24}$. Relative gene expression lelvels for MMP13 and $\beta$-actin as an internal reference gene 


\begin{tabular}{|c|c|c|c|c|c|c|c|}
\hline No & Identity & Case & Common genotype & Heterozygous genotype & Rare genotype & Allelic OR ${ }^{2}(95 \% \mathrm{CI})$ & $P$ \\
\hline \multirow{2}{*}{1} & \multirow{2}{*}{$\begin{array}{c}\text { rs } 11225490 \\
\mathrm{~T}>\mathrm{C}\end{array}$} & ESCC & 0.580 & 0.359 & 0.061 & \multirow{2}{*}{$0.89(0.74-1.08)$} & \multirow{2}{*}{0.251} \\
\hline & & Control & 0.608 & 0.342 & 0.050 & & \\
\hline \multirow{2}{*}{2} & \multirow{2}{*}{$\begin{array}{c}\mathrm{rs} 2252070 \\
\mathrm{G}>\mathrm{A}\end{array}$} & ESCC & 0.369 & 0.454 & 0.177 & \multirow{2}{*}{$0.70(0.60-0.83)$} & \multirow{2}{*}{$2.1 \times 10^{-5}$} \\
\hline & & Control & 0.266 & 0.513 & 0.225 & & \\
\hline \multirow{2}{*}{3} & \multirow{2}{*}{$\begin{array}{l}\mathrm{rs17099788} \\
\mathrm{A}>\mathrm{G}\end{array}$} & ESCC & 0.760 & 0.235 & 0.005 & \multirow{2}{*}{$1.11(0.87-1.41)$} & \multirow{2}{*}{0.393} \\
\hline & & Control & 0.750 & 0.232 & 0.018 & & \\
\hline \multirow{2}{*}{4} & \multirow{2}{*}{$\begin{array}{l}\text { rs3758854 } \\
\text { G }>A\end{array}$} & ESCC & 0.835 & 0.160 & 0.005 & \multirow{2}{*}{$1.09(0.82-1.44)$} & \multirow{2}{*}{0.569} \\
\hline & & Control & 0.827 & 0.163 & 0.010 & & \\
\hline
\end{tabular}

Table 1. Associations between candidate SNPs in the MMP13 and ESCC risk in Jiangsu case-control set (Discovery set). Note: SNP, single nucleotide polymorphism; MMP13, matrix metallopeptidase 13; ESCC, esophageal squamous cell carcinoma; OR, odds ratio; CI, confidence interval. ${ }^{1}$ Data were calculated by logistic regression.

was carried out using the ABI 7500 real-time PCR system in triplicates. The primers used for MMP13 were $5^{\prime}$-GCAAGACTCTCCTGTTCTCAGGAAA- $3^{\prime}$ and $5^{\prime}$-CGGTTACTCCAGATGCTGTATTCAA-3'; and for $\beta$-actin were $5^{\prime}$-GGCGGCACCACCATGTACCCT-3' and 5'-AGGGGCCGGACTCGTCATACT-3'.

Western blotting. KYSE30 and KYSE150 cells were transfected with 20 nM nc RNA or Sp1 siRNAs (siSp1-1 or siSp1-2). Cells were harvested at $48 \mathrm{~h}$ after transfection and cell lysates were immunoblotted as previously reported $^{24,25}$. Antibodies against MMP13 (abcam, ab75606), Sp1 (Millipore, 07-645), or GAPDH (Santa Cruz, 6C5) were used.

Statistical Analyses. Pearson's $\chi^{2}$ test was used to examine the differences in demographic variables, smoking status, drinking status, and genotype distributions of MMP13 SNPs between ESCC cases and healthy controls. Unconditional logistic regression model was utilized to estimate associations between MMP13 genotypes and ESCC risk by odds ratio (OR) and their $95 \%$ confidence intervals (CIs). All ORs were adjusted for age, sex, drinking and smoking status, where it was appropriate. A $P$ value of less than 0.05 was used as the criterion of statistical significance, and all statistical tests were two-sided. All analyses were performed with SPSS software package (Version 16.0, SPSS Inc., Chicago, IL).

\section{Results}

MMP13 rs2252070 is associated with ESCC risk in Chinese Han populations. No Significant differences were found between cases and controls for both case-control sets in terms of median age and sex distribution (both $P>0.05$ ). This indicated that the frequency matching of age and sex was adequate (Supplementary Table 1). The genotype frequencies of MMP13 candidate SNPs (rs11225490 T > C, rs2252070 G > A, rs 17099788 $\mathrm{A}>\mathrm{G}$ and rs3758854 G $>\mathrm{A}$ ) are summarized in Table 1. All observed genotype frequencies in either controls or cases conform to Hardy-Weinberg equilibrium. Distributions of the all genotypes were then compared among patients and controls. Frequencies of rs2252070 genotypes among patients differed significantly from those among controls (all $P<0.05$ ). Logistic regression analyses revealed that rs2252070 SNP was significantly associated with ESCC risk (allelic $\mathrm{OR}=0.70,95 \% \mathrm{CI}=0.60-0.83, P=2.1 \times 10^{-5}$ ) (Table 1). However, no statistically significant differences of other htSNPs were observed between cases and controls (all $P>0.05$ ) (Table 1). As a result, no additional analyses on these SNPs were performed in the next studies.

Associations between genotypes of MMP13 rs2252070 G > A SNP and ESCC risk were calculated using unconditional logistic regression analyses (Table 2). The MMP13 rs2252070 A allele was found to be a protective allele. Individuals with the rs2252070 GA genotype had an OR of $0.65(95 \% \mathrm{CI}=0.49-0.88, P=0.004)$ for developing ESCC in Jiangsu Set, compared with individual having the rs2252070 GG genotype. Similarly, the rs2252070 AA genotypes had a significantly decreased risk for ESCC compared with the rs2252070 GG genotype $(\mathrm{OR}=0.79,95 \% \mathrm{CI}=0.65-0.95, P=0.011)$. In Shandong Set, carriers of the rs2252070 GA or AA genotypes were significantly associated with decreased ESCC risk $\left(\mathrm{OR}=0.65,95 \% \mathrm{CI}=0.53-0.81, P=8.8 \times 10^{-5}\right.$, or $\mathrm{OR}=0.75,95 \% \mathrm{CI}=0.65-0.86, P=4.9 \times 10^{-5}$ ) (Table 3 ). In the pooled analyses, we observed similar results (For GA genotype: $\mathrm{OR}=0.63,95 \% \mathrm{CI}=0.54-0.74, P=1.7 \times 10^{-6}$, For AA genotype: $\mathrm{OR}=0.73,95 \% \mathrm{CI}=0.66-0.81$, $P=1.8 \times 10^{-6}$ ) (Table 2). All ORs were calculated with adjustments of sex, age, smoking and alcohol drinking status. The risk of ESCC associated with the rs2252070 SNP was further investigated by stratifying for age, sex, smoking and alcohol drinking status using the combined data of two case-control sets (Table 3). The variant genotypes of MMP13 rs2252070 (GA or AA) were consistently associated with a significantly decreased risk of ESCC in all subgroups.

MMP13 rs2252070 SNP mediated allele-specific Sp1 binding in ESCC cells. Since rs2252070 SNP is located a potential Sp1 binding sequence of the MMP13 $5^{\prime}$-region (Fig. 1A), we then conducted EMSA to distinguish the differences in binding capacity between the rs2252070G and A alleles to Sp1 (Fig. 1B). As shown in Fig. 1B, we found that Sp1 protein bound only to the biotin-labeled oligonucleotide probe with the $G$ allele but not the A allele probe. We also did competition assay using pure recombinant Sp1 protein and Sp1 antibody (Fig. 1C). Interestingly, there is a super-shift band after adding Sp1 antibody, which conforming that the binding protein with $M M P 13 \mathrm{G}$ probe is $\mathrm{Sp} 1$. 


\begin{tabular}{|c|c|c|c|c|c|}
\hline \multirow[b]{2}{*}{ Studies } & \multicolumn{5}{|c|}{$M M P 13$ rs2252070 G > A } \\
\hline & Genotypes & Cases No. (\%) & Controls No. (\%) & $\mathrm{OR}^{1}(95 \% \mathrm{CI})$ & $P^{1}$ \\
\hline & & $n=588$ & $n=600$ & & \\
\hline \multirow{4}{*}{ Jiangsu set } & GG & $217(36.9)$ & $157(26.6)$ & 1.00 & \\
\hline & GA & $267(45.4)$ & $308(51.3)$ & $0.65(0.49-0.88)$ & 0.004 \\
\hline & AA & $104(17.7)$ & $135(22.5)$ & $0.79(0.65-0.95)$ & 0.011 \\
\hline & & $n=1000$ & $n=1000$ & & \\
\hline \multirow{4}{*}{ Shandong set } & GG & $382(38.2)$ & $274(27.4)$ & 1.00 & \\
\hline & GA & $463(46.3)$ & $513(51.3)$ & $0.65(0.53-0.81)$ & $8.8 \times 10^{-5}$ \\
\hline & AA & $155(15.5)$ & $213(21.3)$ & $0.75(0.65-0.86)$ & $4.9 \times 10^{-5}$ \\
\hline & & $n=1588$ & $n=1600$ & & \\
\hline \multirow{3}{*}{ Pooled } & GG & $599(37.7)$ & $431(26.9)$ & 1.00 & \\
\hline & GA & $730(46.0)$ & $821(51.3)$ & $0.63(0.54-0.74)$ & $1.7 \times 10^{-6}$ \\
\hline & AA & $259(16.3)$ & $348(21.8)$ & $0.73(0.66-0.81)$ & $1.8 \times 10^{-6}$ \\
\hline
\end{tabular}

Table 2. Genotype frequencies of MMP13 rs2252070 genetic variant among patients and controls and their association with ESCC risk. Note: ESCC, esophageal squamous cell carcinoma; MMP13, matrix metallopeptidase 13; OR, odds ratio; CI, confidence interval. ${ }^{1}$ Data were calculated by logistic regression with adjustment for age, sex, smoking and drinking status.

\begin{tabular}{|c|c|c|c|c|c|c|c|c|}
\hline \multirow[b]{2}{*}{ Variable } & \multicolumn{4}{|c|}{$M M P 13$ rs2252070 G $>A$} & \multicolumn{4}{|c|}{$M M P 13$ rs2252070 G $>A$} \\
\hline & $\mathrm{GG}^{1}$ & $\mathbf{G A}^{1}$ & $\mathrm{OR}^{2}(95 \% \mathrm{CI})$ & $P$ & $\mathrm{GG}^{1}$ & $\mathbf{A A}^{1}$ & $\mathrm{OR}^{2}(95 \% \mathrm{CI})$ & $P$ \\
\hline \multicolumn{9}{|l|}{ Sex } \\
\hline Male & $436 / 337$ & $546 / 601$ & $0.71(0.59-0.86)$ & $3.1 \times 10^{-4}$ & $436 / 337$ & $192 / 266$ & $0.76(0.67-0.85)$ & $4.3 \times 10^{-5}$ \\
\hline Female & $163 / 94$ & $184 / 220$ & $0.47(0.33-0.67)$ & $3.2 \times 10^{-5}$ & $163 / 94$ & $67 / 82$ & $0.69(0.56-0.87)$ & 0.001 \\
\hline \multicolumn{9}{|c|}{ Age (years) } \\
\hline$\leq 57$ & $321 / 226$ & $331 / 429$ & $0.56(0.44-0.70)$ & $3.8 \times 10^{-5}$ & $321 / 226$ & $135 / 161$ & $0.78(0.67-0.90)$ & 0.001 \\
\hline$>57$ & $278 / 205$ & $399 / 392$ & $0.77(0.61-0.97)$ & 0.026 & $278 / 205$ & $124 / 187$ & $0.71(0.61-0.82)$ & $6.7 \times 10^{-5}$ \\
\hline \multicolumn{9}{|c|}{ Smoking status } \\
\hline No & $265 / 182$ & $359 / 320$ & $0.76(0.59-0.96)$ & 0.024 & $265 / 182$ & $131 / 143$ & $0.81(0.69-0.94)$ & 0.007 \\
\hline Yes & $334 / 249$ & $371 / 501$ & $0.55(0.45-0.69)$ & $5.9 \times 10^{-6}$ & $334 / 249$ & $128 / 205$ & $0.69(0.60-0.79)$ & $7.8 \times 10^{-6}$ \\
\hline \multicolumn{9}{|c|}{ Drinking status } \\
\hline No & $309 / 206$ & $413 / 420$ & $0.65(0.52-0.81)$ & $1.8 \times 10^{-4}$ & $309 / 206$ & $131 / 179$ & $0.70(0.60-0.81)$ & $9.0 \times 10^{-6}$ \\
\hline Yes & $290 / 225$ & $317 / 401$ & $0.62(0.49-0.77)$ & $3.2 \times 10^{-5}$ & $290 / 225$ & $128 / 169$ & $0.77(0.67-0.89)$ & $3.7 \times 10^{-4}$ \\
\hline
\end{tabular}

Table 3. Risk of ESCC associated with MMP13 rs2252070 G > A genotypes by age, sex, smoking status and drinking status. Abbreviations: ESCC, esophageal squamous cell carcinoma; MMP13, matrix metallopeptidase 13; OR, odds ratio; CI, confidence interval. ${ }^{1}$ Number of case patients with genotype/number of control subjects with genotype. ${ }^{2}$ Data were calculated by logistic regression, adjusted for sex, age, smoking, and drinking status, where it was appropriate.

Allelic regulation of rs2252070 on MMP13 promoter activities. Because the rs 2252070 SNP is located in the Sp1 binding sit of MMP13 promoter, we speculated that this polymorphism will influence gene expression of $M M P 13$. Therefore, we examined the promoter activity of this region by two luciferase reporter constructs (Fig. 2A) with different rs2252070 allele in ESCC KYSE30 and KYSE150 cells. Interestingly, the MMP13 rs2252070G allelic reporter construct (pMMP-G) showed significantly higher luciferase activities compared to the rs2252070A allelic reporter construct (pMMP-A) in KYSE30 cells $(P<0.01)$ (Fig. 2B). Moreover, KYSE150 cells transfected with pMMP-G showed significantly higher luciferase activities compared to cells expressing pMMP-A $(P<0.05)$ (Fig. 2C). This indicates that Sp1 could bind the rs2252070G allelic MMP13 promoter and prompt increased $M M P 13$ expression.

Impacts of rs2252070 on MMP13 expression in vivo. Considering rs2252070 G-to-A change could impact MMP13 promoter activity in cancer cells, we investigated whether there is an allele-specific effect of rs2252070 SNP on MMP13 expression in esophagus tissue specimens (Fig. 3). We found that there were significantly lower MMP13 mRNA levels (mean \pm SD) among carriers of the rs2252070 AA genotype compared to carriers of the GG genotype in ESCC tissues $(0.014 \pm 0.026[n=12]$ vs. $0.119 \pm 0.074[n=24], P<0.01)$ or normal esophagus tissues $(0.024 \pm 0.034[n=12]$ vs. $0.088 \pm 0.050[n=24], P<0.01)$. Similar results have also been observed for GA genotype in ESCC tissues $(0.072 \pm 0.058[n=30]$ vs. $0.119 \pm 0.074[n=24], P<0.05)$ or normal esophagus tissues $(0.060 \pm 0.043[n=30]$ vs. $0.088 \pm 0.050[n=24], P<0.05)$. 
A AliBaba2. 1 predicts the following sites in your sequence

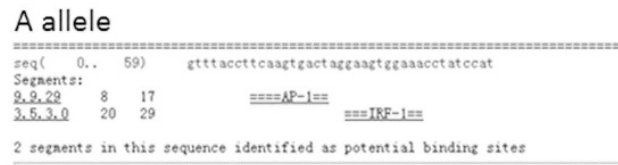

B

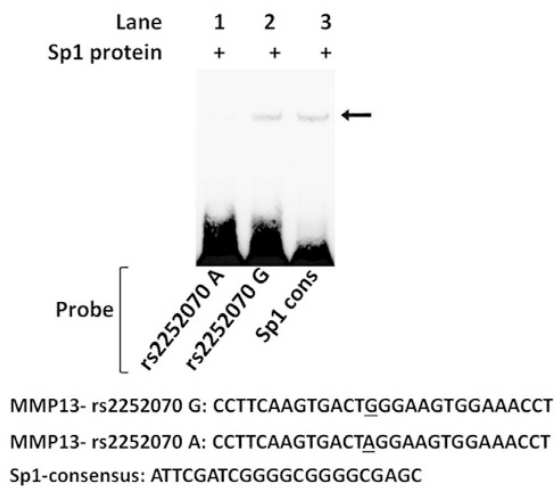

G allele

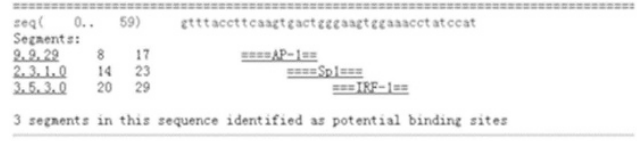

C

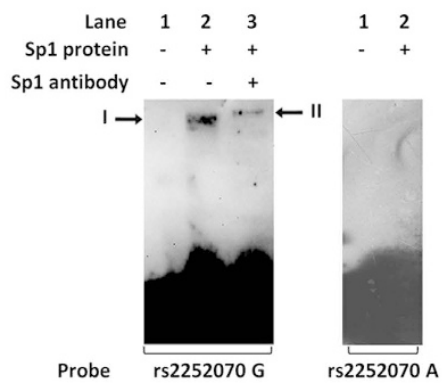

Figure 1. Abolishment of a Sp1 binding site in the MMP13 promoter by the rs2252070 G $>$ A genetic variant. (A) Alibaba 2.1 prediction. (B) Electrophoretic mobility-shift assay (EMSA) with biotin-labeled rs2252070G or rs2252070A probes and KYSE30 nuclear extracts. Lanes 1 and 6, probe only; lanes 3 and 8, probe and nuclear extracts; lanes 2 and 7, probe and nuclear extracts plus 100× unlabeled rs2252070G; lanes 4 and 9, probe and nuclear extracts plus $100 \times$ unlabeled rs2252070A; lanes 5 and 10, probe and nuclear extracts plus $100 \times$ unlabeled Sp1 consensus probes. (C) Super-EMSA with biotin-labeled rs2252070G or rs2252070A probes, pure recombinant Sp1 protein or Sp1 antibody. Lanes 1, probe only; lanes 2, probe and recombinant Sp1 protein; lanes 3, probe, recombinant Sp1 protein and Sp1 antibody.

A

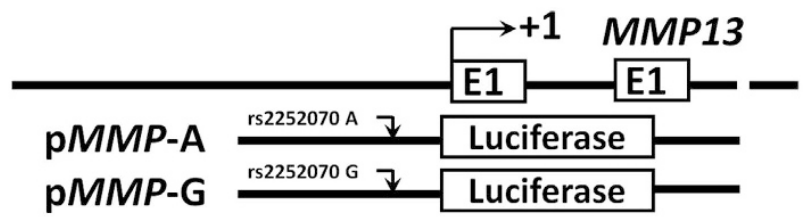

B

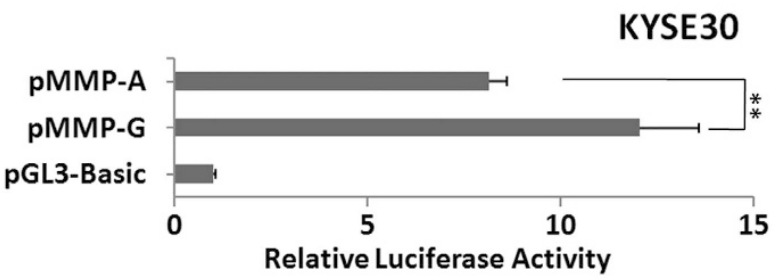

C

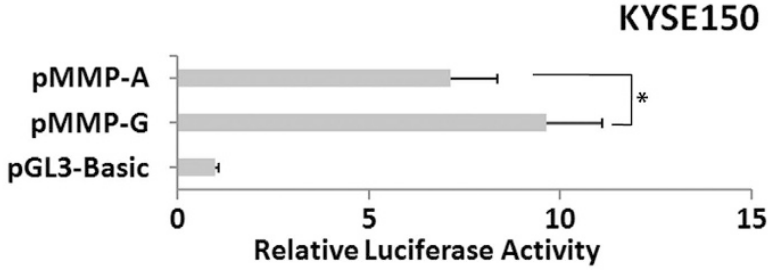

Figure 2. Transient luciferase reporter gene expression assays with constructs containing different rs2252070 alleles of MMP13 5'-region (A) in KYSE30 cells (B) or KYSE150 cells (C). To standardize transfection efficiency, we cotransfected pRL-SV40 with these reporter gene constructs. Fold-changes were calculated by defining the luciferase activity of cells co-transfected with pGL3-basic as 1. All experiments were performed in triplicates in three independent transfection experiments and each value represents mean \pm SD. Compared with pGL3-Basic transfected cells, $* P<0.05$; $* * P<0.01$. 
A

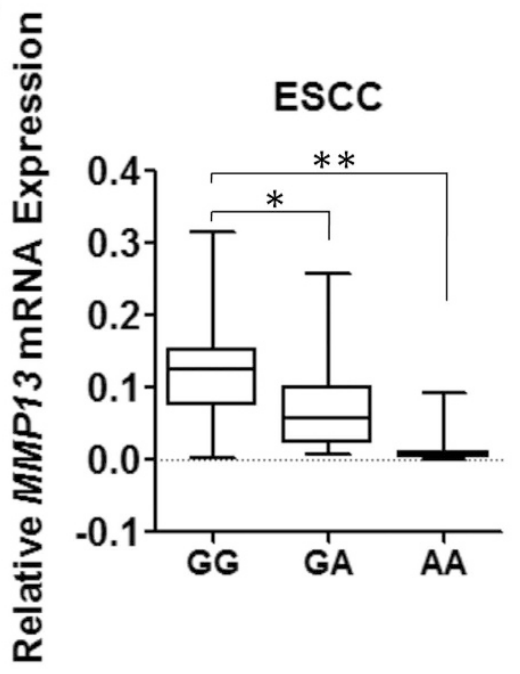

B

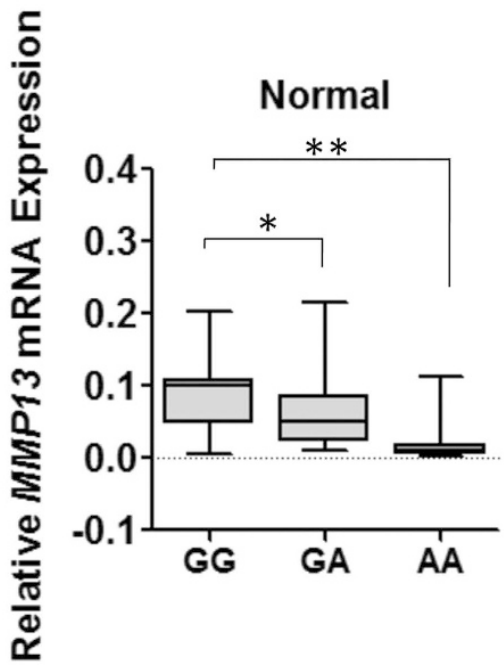

Figure 3. MMP13 mRNA expression (mean $\pm \mathrm{SD}$ ) in normal and cancerous esophageal tissues grouped by MMP13 rs2252070 genotypes. The expression of individual $M M P 13 \mathrm{mRNA}$ was calculated relative to expression of $\beta$-actin using the $2^{-\mathrm{dCt}}$ method. $* P<0.05$; $* * P<0.01$.

A

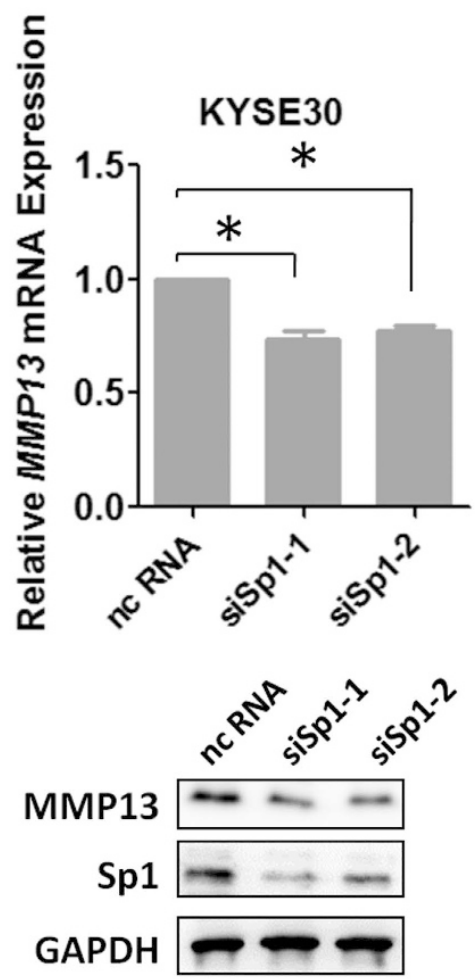

B
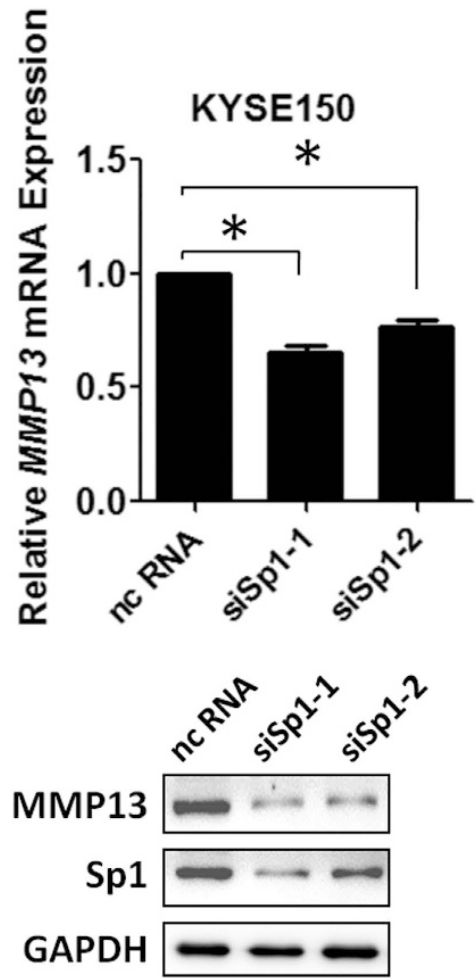

Figure 4. Silencing Sp1 expression repressed MMP13 expression in KYSE30 and KYSE150 cells. The expression of individual MMP13 mRNA was calculated relative to expression of $\beta$-actin. $* P<0.05$. Protein expression of MMP13, Sp1 and GAPDH was detected using Western Blotting.

To further verify if $\mathrm{Sp} 1$ indeed promotes MMP13 expression, we knocked-down endogenous Sp1 with siRNAs and examine the expression of MMP13 in two ESCC cell lines. Decreased MMP13 expression was observed after Sp1 silencing (Fig. 4), suggesting that Sp1 enhance endogenous MMP13 expression in ESCC cells. 


\section{Discussion}

In the current study, we systematically examined the impacts of SNPs in the MMP13 locus on ESCC susceptibility via a case-control design as well as gene expression of MMP13 in vitro and in vivo. After genotyping 4 htSNPs at the discovery stage, we identified one ESCC susceptibility genetic polymorphism (rs2252070) which were validated in another case-control sets. Reporter gene assays indicated that the ESCC susceptibility SNP rs2252070 a potential MMP13 promoter has a genotype-specific effect on MMP13 expression. Our observations support the hypothesis that genetic polymorphisms in oncogene regulatory elements might impact genetic susceptibility of ESCC.

ESCC frequently shows extensive local invasion or regional lymph node metastasis at diagnosis and, thus, is one of the most common aggressive diseases with poor outcome. Tumor invasion and metastasis require the actions of MMPs for degradation of extracellular matrix. MMP13, a well-known zinc-dependent MMP collagenase, has been identified as a essential MMP in facilitating ESCC development. Elevated MMP13 expression was not only observed in ESCC tissues but also associated with tumor invasion, vascular permeation, and lymph node metastasis $^{12-15}$. Therefore, it is crucial to examine fine-regulation of $M M P 13$ expression during esophageal carcinogenesis. Accumulated evidences demonstrated that the MMP13 rs2252070 polymorphism is a regulatory polymorphism in cells, but the detailed mechanisms are far from clear. Here, for the first time, we found that the rs2252070 G allele but not A allele could bind Sp1 and promote MMP13 expression in ESCC. This is consistent with our molecular epidemiology studies showing that MMP13 rs2252070 A allele is a protecting allele of ESCC in Chinese. That is, subjects carrying the MMP13 rs2252070 A allele without Sp1 binding have less oncogene $M M P 13$ expression; therefore, these carriers show decreased risk to develop ESCC.

In all, we demonstrated that functional MMP13 rs2252070 SNP was associated with a significantly decreased risk of ESCC in Chinese Han populations. Functional analysis showed that the rs2252070A allele contributes to significantly decreased expression of MMP13 in vitro and in vivo in the target tissues, which is most likely due to a diminished Sp1 regulation. These findings constitute strong evidence in support of the notion that MMP13 might function as a key oncogene in esophageal carcinogenesis.

\section{References}

1. Jemal, A. et al. Cancer statistics, 2010. CA Cancer J Clin 60, 277-300 (2010).

2. Gao, Y. T. et al. Risk factors for esophageal cancer in Shanghai, China. I. Role of cigarette smoking and alcohol drinking. Int J Cancer 58, 192-196 (1994).

3. Hu, J. et al. Risk factors for oesophageal cancer in northeast China. Int J Cancer 57, 38-46 (1994).

4. Cui, R. et al. Functional variants in ADH1B and ALDH2 coupled with alcohol and smoking synergistically enhance esophageal cancer risk. Gastroenterology 137, 1768-1775 (2009).

5. Abnet, C. C. et al. A shared susceptibility locus in PLCE1 at 10q23 for gastric adenocarcinoma and esophageal squamous cell carcinoma. Nat Genet 42, 764-767 (2010).

6. Wang, L. D. et al. Genome-wide association study of esophageal squamous cell carcinoma in Chinese subjects identifies susceptibility loci at PLCE1 and C20orf54. Nat Genet 42, 759-763 (2010).

7. Wu, C. et al. Genome-wide association study identifies three new susceptibility loci for esophageal squamous-cell carcinoma in Chinese populations. Nat Genet 43, 679-684 (2011).

8. Abnet, C. C. et al. Genotypic variants at $2 \mathrm{q} 33$ and risk of esophageal squamous cell carcinoma in China: a meta-analysis of genomewide association studies. Hum Mol Genet 21, 2132-2141 (2012).

9. Wu, C. et al. Genome-wide association analyses of esophageal squamous cell carcinoma in Chinese identify multiple susceptibility loci and gene-environment interactions. Nat Genet 44, 1090-1097 (2012).

10. Wu, C. et al. Joint analysis of three genome-wide association studies of esophageal squamous cell carcinoma in Chinese populations. Nat Genet 46, 1001-6 (2014).

11. Deryugina, E. I. et al. Matrix metalloproteinases and tumor metastasis. Cancer Metastasis Rev 25, 9-34 (2006).

12. Hu, Y. C. et al. Profiling of differentially expressed cancer-related genes in esophageal squamous cell carcinoma (ESCC) using human cancer cDNA arrays: overexpression of oncogene MET correlates with tumor differentiation in ESCC. Clin Cancer Res 7, 3519-3525 (2001).

13. Etoh, T. et al. Increased expression of collagenase-3 (MMP-13) and MT1-MMP in oesophageal cancer is related to cancer aggressiveness. Gut 47, 50-56 (2000).

14. Gu, Z. D. et al. Matrix metalloproteinases expression correlates with survival in patients with esophageal squamous cell carcinoma. Am J Gastroenterol 100, 1835-1843 (2005).

15. Ye, Q. et al. MUC1 induces metastasis in esophageal squamous cell carcinoma by upregulating matrix metalloproteinase 13. Lab Invest 91, 778-787 (2011).

16. Yoon, S. et al. MMP13 promoter polymorphism is associated with atherosclerosis in the abdominal aorta of young black males. Matrix Biol 21, 487-498 (2002).

17. Li, H. et al. Association of matrix metalloproteinase family gene polymorphisms with lung cancer risk: logistic regression and generalized odds of published data. Sci Rep 5, 10056 (2015).

18. Gao, P. et al. Common polymorphism in the MMP-13 gene may contribute to the risk of human cancers: a meta-analysis. Tumour Biol 35, 10137-10148 (2014).

19. Moreno-Ortiz, J. M. et al. Association of MMP7-181A/G and MMP13-77A/G polymorphisms with colorectal cancer in a Mexican population. Genet Mol Res 13, 3537-3544 (2014).

20. VAN Nguyen, S. et al. Gene polymorphism of matrix metalloproteinase-12 and -13 and association with colorectal cancer in Swedish patients. Anticancer Res 33, 3247-3250 (2013)

21. Pan, W. et al. Functional BCL-2 regulatory genetic variants contribute to susceptibility of esophageal squamous cell carcinoma. Sci Rep 5, 11833 (2015).

22. Sun, T. et al. Haplotypes in matrix metalloproteinase gene cluster on chromosome 11q22 contribute to the risk of lung cancer development and progression. Clin Cancer Res 12, 7009-7017 (2006).

23. Pan, W. et al. A functional lncRNA HOTAIR genetic variant contributes to gastric cancer susceptibility. Mol Carcinog 55, $90-96$ (2015).

24. Wang, X. et al. Silencing of long noncoding RNA MALAT1 by miR-101 and miR-217 inhibits proliferation, migration, and invasion of esophageal squamous cell carcinoma cells. J Biol Chem 290, 3925-3935 (2015).

25. Pan, W. et al. Whole exome sequencing identifies lncRNA GAS8-AS1 and LPAR4 as novel papillary thyroid carcinoma driver alternations. Hum Mol Genet doi: 10.1093/hmg/ddw056 (2016). 


\section{Acknowledgements}

This study was financially supported by National Natural Science Foundation of China (31271382 \& 81160249); the Fundamental Research Funds for the Central Universities (YS1407); the National High-Tech Research and Development Program of China (2015AA020950); the open project of State Key Laboratory of Molecular Oncology (SKL-KF-2015-05); Innovation and Promotion Project of Beijing University of Chemical Technology.

\section{Author Contributions}

M.Y. and L.Z. conceived and designed the experiments; M.S. performed the experiments; M.S., J.X. and H.X. analyzed the data; W.Y., J.X., X.X., W.P., S.H., J.S., C.Z. and H.X. contributed materials/analysis tools; M.Y. and L.Z. wrote the manuscript. All authors reviewed and approved the manuscript prior to submission.

\section{Additional Information}

Supplementary information accompanies this paper at http://www.nature.com/srep

Competing financial interests: The authors declare no competing financial interests.

How to cite this article: Shi, M. et al. The Sp1-mediaded allelic regulation of MMP13 expression by an ESCC susceptibility SNP rs2252070. Sci. Rep. 6, 27013; doi: 10.1038/srep27013 (2016).

(c) (i) This work is licensed under a Creative Commons Attribution 4.0 International License. The images or other third party material in this article are included in the article's Creative Commons license, unless indicated otherwise in the credit line; if the material is not included under the Creative Commons license, users will need to obtain permission from the license holder to reproduce the material. To view a copy of this license, visit http://creativecommons.org/licenses/by/4.0/ 